\title{
Metformin does not prevent atherosclerotic cardiovascular disease
}

\author{
Craig D Williams* \\ Oregon State University/ OHSU College of Pharmacy, USA
}

\begin{abstract}
The recent description of the mechanism of action of metformin at the cellular level offers the opportunity to revisit the question of whether or not metformin is beneficial in patients with atherosclerotic cardiovascular disease (ASCVD). In the context of newer, larger trials which examined the role of glycemic management in high risk patients with diabetes, the landmark UK Prospective Diabetes Study (UKPDS) and University Group Diabetes Program (UGDP) study are revisited. A close re-examination of these trials reveals that the apparent finding of ASCVD benefit for metformin in the UKPDS is likely the result of trial design rather than an antiatherogenic benefit from metformin.
\end{abstract}

The recent description of the cellular mechanism of action of metformin offers the opportunity to re-visit the question of whether or not this drug has any beneficial effect on atherosclerotic cardiovascular disease (ASCVD)[1] In the 2012 consensus statement on managing hyperglycemia, the American Diabetes Association (ADA) and European Association for the Study of Diabetes (EASD) state that metformin "may have some cardiovascular benefits and would appear to be a useful drug in the setting of CAD." [2] This opinion is popular in clinical practice. The single citation offered by ADA/EASD in support of a cardiovascular benefit of metformin is the UK Prospective Diabetes Study (UKPDS) [3].But the UKPDS was a complicated trial with varying degrees of glycemic control across multiple substudies [3-6]. The results are also now nearly 2 decades old and our understanding of the impact of glycemic control on atherosclerosis has evolved. It is thus fair to ask again whether or not metformin has any antiatherogenic properties aside from its antihyperglycemic effects.

The UKPDS began recruiting patients in 1977, just several years after the publication of the University Group Diabetes Program trial (UGDP). The UGDP trial was specifically designed to study the vascular effects of antihyperglycemic therapies in patients with adultonset diabetes [7]. In addition to tolbutamide and insulin, UGDP used phenformin, a biguanide similar to metformin. In a surprise finding, the phenformin arm was stopped early when a preliminary analysis of study data showed a fourfold increase in cardiovascular death compared to placebo $(12.7 \%$ vs. $3.1 \%, p=0.04)$ [8] Compared to the insulin arms of the trial, phenformin had a nearly twofold increase in cardiovascular death although this finding was not statistically significant $(12.7 \%$ vs. $6.8 \%, \mathrm{p}=0.11$ ) [8].

The adverse cardiovascular outcomes with phenformin were an understandable cause of some concern for metformin. Thus when UKPDS began recruiting just six years after the publication of the phenformin findings of UGDP, it did so with a complicated protocol involving two different metformin substudies, one of monotherapy and one as a sulfonylurea add-on.

The monotherapy trial enrolled only overweight patients $(n=1,704$ with 342 assigned metformin) while the sulfonylurea add-on arm enrolled the $13 \%$ of the study population who failed to achieve their "tight" glycemic goal on sulfonylurea monotherapy (n=567 with 268 assigned metformin).[3] The findings of these substudies was that metformin monotherapy was associated with a $39 \%$ reduction in myocardial infarction ( $\mathrm{p}=0.01$ ) and a $36 \%$ reduction in all-cause mortality $(\mathrm{p}=0.01)$ while metformin add-on therapy was associated with a $60 \%$ increase in all-cause mortality ( $\mathrm{p}=0.04)$ including a non-significant $79 \%$ increase in fatal myocardial infarction $(11.0 \%$ vs. $6.2 \%, \mathrm{p}=0.14)$.

So, a combined analysis of the clinical endpoints of the UGDP and UKPDS suggests that in patients with adult-onset diabetes, phenformin increases CV death while metformin increases mortality when added to a sulfonylurea but reduces mortality and myocardial infarction when used as monotherapy in overweight patients. Given these apparent contradictions, does the new understanding of the mechanism of action of metformin offer any insight into whether it could be anti-atherogenic, pro-atherogenic or both?

In an elegant series of experiments published in 2014, metformin was found to inhibit a mitochondrial enzyme in hepatocytes which affects the ratio of cytosolic NADH-NAD [9]. This results in an inhibition of the conversion of lactate to pyruvate which subsequently impairs hepatic gluconeogenesis. Could this molecular mechanism plausibly affect the initiation or progression of ASCVD?

Atherosclerosis is well understood to be a process of vascular inflammation in response to the retention of atherogenic lipoprotein particles in the artery wall [10]. While patients with diabetes are well known to have a higher rate of ASCVD events compared to patients without diabetes, the etiology is this greater atherosclerotic burden is multifactorial and the role of hyperglycemia remains controversial [11]. However, a drug which inhibits a hepatic enzyme to reduce gluconeogenesis would not seem to offer any direct benefit on the

Correspondence to: Craig D Williams, Oregon State University/ OHSU College of Pharmacy, 2730 SW Moody Ave, CL5CP, Portland, OR 97201, USA, Tel: 503.494.1598; Fax: 503.494.8797; E-mail: williacr@ohsu.edu

Received: August 22, 2017; Accepted: September 15, 2017; Published: September 19, 2017 
known processes of atherosclerosis. While it is possible that additional mechanisms of action will be discovered for metformin, that is unlikely given the elegant experiments that led to the current discovery [1].

If the mechanism of action of metformin makes it unlikely that it offers any direct antiatherogenic benefit, what might explain the apparent benefit in the monotherapy arm of the UKPDS?

Importantly, the metformin monotherapy arm of UKPDS compared intensive therapy with metformin to conventional therapy with diet [3]. Two important aspects of this design could have falsely accounted for the apparent ASCVD benefit of metformin.

First, glycemic control was improved with "intensive" metformin therapy (median daily dose $2550 \mathrm{mg}$ ) compared to the conventional dietary group. During the first 5 years of follow-up, A1C averaged $6.7 \%$ in the metformin group compared to $7.5 \%$ in the conventional group [3]. Over the 10 years of follow-up, A1C averaged 7.5\% in the metformin group compared to $8.0 \%$ in the conventional group. While these differences are not large, they should not be discounted. Recent trials which have examined the relationship between glycemic control and ASCVD have found consistent trends for reductions in ASCVD events with reductions in A1C [11-15]. In the ACCORD trial (Action to Control Cardiovascular Risk in Diabetes Study Group), an A1C of $6.4 \%$ compared to $7.5 \%$ was associated with a non-significant $10 \%$ risk reduction in the composite endpoint of non-fatal MI, non-fatal stroke and CVD death and a significant $24 \%$ risk reduction in non-fatal MI [12].

Second, while there were no major hypoglycemic episodes in the metformin monotherapy arm of UKPDS, the conventional comparator group had a rate of major hypoglycemic episodes of $0.7 \%$ per year [3]. While it may seem implausible that a dietary control group would have episodes of major hypoglycemia, a closer look at the design of UKPDS reveals that $44 \%$ of that cohort could not meet their glycemic goal on diet alone and received supplemental therapies, primarily with sulfonylureas and insulin [3].These are agents which are well known to carry a risk of major hypoglycemia.

Increased mortality risk attributable to hypoglycemia is an area of increasing interest among the diabetes community [16-19]. In the newer trials which examined the relationship between glycemic control and clinical outcomes, trends for total mortality are increased despite trends for reductions in cardiovascular events $[11,15]$. Strong associations have been found between episodes of hypoglycemia and mortality, a relationship which may be explained by arrhythmias and sudden cardiac death $[16,19]$.

Last, it should be noted that the pharmacokinetics of metformin also argue against a direct ASCVD benefit. While metformin is found in high concentrations in the gut wall and liver, it is found in very small concentrations in the plasma and other tissue, including cardiac and vascular [20].

So while the newly described mechanism of action of metformin does not appear to offer an explanation for the apparent ASCVD benefits seen in the monotherapy arm of the UKPDS, the complicated design of that trial itself does offer an explanation. In the context of our current understanding of the relationships between glycemic control, atherosclerosis and mortality, improving glycemic control without any major episodes of hypoglycemia could well account for the apparent benefit of metformin in the monotherapy arm of UKPDS. A lack of direct ASCVD benefit from metformin would also explain why benefits were not seen in the metformin add-on arm in UKPDS. So while metformin remains a great first line choice for glycemic control in most of our patients with diabetes, it is time to stop thinking about it as an antiatherogenic drug.

\section{References}

1. Ferrannini E (2014) The target of metformin in type 2 diabetes. N Engl J Med 371: 1547-1548. [Crossref]

2. Inzucchi SE, Bergenstal RM, Buse JB, Diamant M, Ferrannini E, et al. (2012) Management of hyperglycemia in type 2 diabetes: a patient-centered approach: position statement of the American Diabetes Association (ADA) and the European Association for the Study of Diabetes (EASD). Diabetes care 35: 1364-1379. [Crossref]

3. Effect of intensive blood-glucose control with metformin on complications in overweight patients with type 2 diabetes (UKPDS 34). UK Prospective Diabetes Study (UKPDS) Group. (1998) Lancet 352: 854-865.[Crossref]

4. Intensive blood-glucose control with sulphonylureas or insulin compared with conventional treatment and risk of complications in patients with type 2 diabetes (UKPDS 33). UK Prospective Diabetes Study (UKPDS) Group. (1998) Lancet 352: 837-853. [Crossref]

5. Efficacy of atenolol and captopril in reducing risk of macrovascular and microvascular complications in type 2 diabetes: UKPDS 39. UK Prospective Diabetes Study Group. (1998) $B M J 317,713-720$. [Crossref]

6. Tight blood pressure control and risk of macrovascular and microvascular complications in type 2 diabetes: UKPDS 38. UK Prospective Diabetes Study Group. (1998) BMJ 317: 703-713. [Crossref]

7. Goldner MG, Knatterud GL, Prout TE (1971) Effects of hypoglycemic agents on vascular complications in patients with adult-onset diabetes. 3. Clinical implications of UGDP results. JAMA 218: 1400-1410. [Crossref]

8. Knatterud GL, Meinert CL, Klimt CR, Osborne RK, Martin DB (1971) Effects of hypoglycemic agents on vascular complications in patients with adult-onset diabetes. IV. A preliminary report on phenoformin results. JAMA 217: 777-784 .[Crossref]

9. Madiraju AK, Erion DM, Rahimi Y, Zhang XM, Braddock DT, et al. (2014) Metformin suppresses gluconeogenesis by inhibiting mitochondrial glycerophosphate dehydrogenase. Nature 510: 542-546 [Crossref]

10. Tabas I, Williams KJ, Boren J (2007) Subendothelial lipoprotein retention as the initiating process in atherosclerosis: update and therapeutic implications. Circulation 116: 1832-1844. [Crossref]

11. Jay S Skyler, Richard Bergenstal, Robert O. Bonow, John Buse, Prakash Deedwania et al. (2009) Intensive glycemic control and the prevention of cardiovascular events: implications of the ACCORD, ADVANCE, and VA diabetes trials: a position statement of the American Diabetes Association and a scientific statement of the American College of Cardiology Foundation and the American Heart Association. Diabetes care 32: 187-192. [Crossref]

12. Action to Control Cardiovascular Risk in Diabetes Study, G., et al. (2008) Effects of intensive glucose lowering in type 2 diabetes. N Engl J Med 358: 2545-2559. [Crossref]

13. ADVANCE Collaborative Group, Patel A, MacMahon S, Chalmers J, Neal B, et al. (2008) Intensive blood glucose control and vascular outcomes in patients with type 2 diabetes. N Engl J Med 358: 2560-2572. [Crossref]

14. Duckworth W, Abraira C, Moritz T, Reda D, Emanuele N, et al. (2009) Glucose control and vascular complications in veterans with type 2 diabetes. $N$ Engl J Med 360: 129139. [Crossref]

15. Riddle MC, Ambrosius WT, Brillon DJ, Buse JB, Byington RP et al. (2010) Epidemiologic relationships between $\mathrm{A} 1 \mathrm{C}$ and all-cause mortality during a median 3.4-year follow-up of glycemic treatment in the ACCORD trial. Diabetes care 33: 983-990. [Crossref]

16. Chow E, Bernjak A, Williams S, Fawdry RA, Hibbert S, et al. (2014) Risk of cardiac arrhythmias during hypoglycemia in patients with type 2 diabetes and cardiovascular risk. Diabetes 63: 1738-1747. [Crossref]

17. Dandona P, Chaudhuri A, Dhindsa S (2010) Proinflammatory and prothrombotic effects of hypoglycemia. Diabetes Care 33: 1686-1687. [Crossref]

18. Desouza CV, Bolli GB, Fonseca V (2010) Hypoglycemia, diabetes, and cardiovascular events. Diabetes care 33, 1389-1394. [Crossref]

19. Zoungas S, Patel A, Chalmers J, de Galan BE, Li Q, et al. (2010) Severe hypoglycemia and risks of vascular events and death. N Engl J Med 363: 1410-1418. [Crossref]

20. Graham GG, Punt J, Arora M, Day RO, Doogue MP, et al. (2011) Clinical pharmacokinetics of metformin. Clin Pharmacokinet 50: 81-98. [Crossref]

Copyright: (C2017 Williams CD. This is an open-access article distributed under the terms of the Creative Commons Attribution License, which permits unrestricted use, distribution, and reproduction in any medium, provided the original author and source are credited. 